\title{
A FREE BOUNDARY PROBLEM CONNECTED WITH THE OPTIMAL STOPPING PROBLEM FOR DIFFUSION PROCESSES
}

BY

\section{DANIE L B. KOT LOW}

ABSTRACT. This paper deals with a free boundary problem for a parabolic equation in one space variable which arises from the problem of selecting an optimal stopping strategy for the diffusion process connected with the equation. It is shown that a solution of the free boundary problem yields the solution of a minimum problem concerning supersolutions of the parabolic equation as well as the solution of the oprimal stopping problem. Theorems regarding the existence, uniqueness, regularity, and approach to the steady state of solutions of the free boundary problem are established.

1. Introduction. Let

$$
L_{0} \equiv d(x) \frac{\partial^{2}}{\partial x^{2}}+b(x) \frac{\partial}{\partial x}+d(x)
$$

where $a, b, c \in C^{2+a}([0, \infty))$ for some $a \in(0,1), a(x) \geq a_{0}>0(0 \leq x<\infty), c(x)$ $\leq 0(0 \leq x<\infty)$, and let $L$ be the parabolic operator $L=L_{0}-\partial / \partial t$. Let $\psi \epsilon$ $C^{3}([0, \infty))$ be a given real-valued function such that, for some $d>0$,

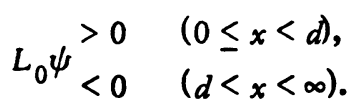

In this paper, we treat the following

Free boundary problem. Determine a curve $x=s(t)(0 \leq t<\infty)$ such that $s(t)>0$, together with a solution $u$ of the parabolic equation

$$
L u=0
$$

in the (unknown) region $C$ defined by

$$
C=\{(x, t) ; 0<x<s(t), 0<t<\infty\},
$$

which satisfies the conditions

$$
\begin{array}{ll}
u(x, t) \geq \psi(x), & (x, t) \in C, \\
u(0, t)=\psi(0), & 0 \leq t<\infty,
\end{array}
$$

Received by the editors September 8, 1972.

AMS (MOS) subject classifications (1970). Primary 35K60; Secondary 62C99, 93E20.

Key words and phrases. Free boundary problem, parabolic equation, minimal supersolution, optimal stopping, diffusion process, optimal stochastic control. 


$$
\begin{aligned}
u(x, 0) & =\psi(x), \quad 0 \leq x \leq s(0), \\
u(s(t), t) & =\psi(s(t)), \quad 0 \leq t<\infty, \\
(\partial u / \partial x)(s(t), t) & =\psi^{\prime}(s(t)), \quad 0 \leq t<\infty .
\end{aligned}
$$

Condition (1.3) will determine the value of $s(0)$; then the extra boundary condition will serve to determine uniquely the entire unknown portion of the boundary of $C$.

Our free boundary problem arises in connection with the following optimization problem in the theory of stochastic processes. Let $X(t)(t \leq 0)$ be a diffusion process on the nonnegative real axis with absorption at $x=0$ and stopping at $t=0$. Suppose the Dynkin generator of this process agrees on $(0, \infty)$ with the differential operator $L_{0}$ on smooth functions. Let $\bar{M}$ be the set of all Markov times (stopping times) $\bar{m}$ for this process. We wish to evaluate the function

$$
v(x, t)=\sup _{\bar{m} \in \bar{M}} E_{X(-t)=x}(\psi(X(\bar{m})))
$$

and to find a Markov time $\bar{m}_{\text {opt }} \in \bar{M}$ such that

$$
E_{X(-t)=x}\left(\psi\left(X\left(\bar{m}_{\mathrm{opt}}\right)\right)\right)=v(x, t) .
$$

If we think of the elements of $\bar{M}$ as stopping strategies, the problem is to find a strategy which will maximize the expected value of the payoff function $\psi$ at the point of stopping, independently of the starting condition $X(-t)=x(0 \leq t<\infty)$. A strategy $\bar{m}_{\text {ope }}$ which does this is called an optimal stopping time. It turns out that if we solve the free boundary problem (1.2)-(1.7) and set

$$
\hat{u}(x, t)= \begin{cases}u(x, t) & \text { if }(x, t) \in C \\ \psi(x) & \text { if }(x, t) \in \bar{Q}-C\end{cases}
$$

where $Q=(0, \infty) \times(0, \infty)$, then $v \equiv \hat{u}$ and we may choose

$$
\bar{m}_{\mathrm{opt}}=\inf \{t ;(X(t),-t) \notin C\} .
$$

I.e., the optimal strategy is to continue as long as the graph of position vs. time remaining lies in the continuation region $C$ and to stop as soon as it leaves this region. The simple form of the optimal strategy is a consequence of the assumption (1.1) on the form of the payoff function, which has the interpretation that it pays to continue on a purely local basis when the sample path is in $(0, d)$ but it costs on a local basis when the sample path is in $(d, \infty)$. This interpretation may be read off from Dynkin's formula 


$$
E_{x}(\psi(X(\bar{m})))=\psi(x)+E_{x}\left(\int_{0}^{\bar{m}}\left(L_{0} \psi\right)(X(t)) d t\right)
$$

by setting

$$
\bar{m}=\delta \wedge \inf \{t ;|X(t)-x| \geq \delta\} \text { for small } \delta>0
$$

The connection between optimal stopping and free boundary problems was noticed by Chernoff [1] and McKean [13], among others, in special cases, and was explored in detail by Grigelionis and Širjaev [9] for general homogeneous Markov processes. We refer the reader to the survey papers of Chernoff [2] and Grigelionis [8] for an overview of the subject of optimal stopping problems and for a more extensive bibliography. The basic facts about optimal stopping may be found in Dynkin [3], [4] and Dynkin and Juškevič [5]. A number of free boundary problems which are similar to (1.2)-(1.7) have been treated by Ventcel' [19], Thi [18], Kruzhkov [12] and Sackett [15]. Kolodner [11], McKean [13] and Sherman [17] have reduced free boundary problems of optimal stopping type to nonlinear integral equations, but these have (so far) proved unamenable to solution. Also, Sackett [16] has solved numerically a free boundary problem which arises from an optimal stopping problem.

In $\$ 2$, we assume that we have in hand a solution $\{s, u\}$ of (1.2)-(1.7) which has certain regularity and we establish a number of its properties. The main result (Theorem 1 ) is that, if $\hat{u}$ is defined by (1.8), then $\hat{u}$ is the least of all L-supersolutions $v$ on $\bar{Q}$ such that $v \geq \psi$ on $\bar{Q}$. This leads immediately to a uniqueness theorem (Theorem 2 ) for the free boundary problem. Next, we establish the connection described above between the free boundary problem and the optimal stopping problem (Theorem 3). Then we solve the steady state analogue of (1.2)(1.7) (Theorem 4), which corresponds in the probabilistic interpretation to the case where the process is started at $t=-\infty$ (i.e., it is allowed to continue indefinitely until absorption occurs), and we establish the convergence of the solution of (1.2)-(1.7) to the steady state solution as $t \rightarrow \infty$ (Theorem 5). Finally, in $\$ 3$ we construct the solution of the free boundary problem by an adaptation of the method of E. Röthe [14]. Here we make the mild technical assumption that

$$
\left(L_{0} \psi\right)(x) \leq-c_{0}(x-d)^{\beta} \quad(x \geq d)
$$

where $c_{0}$ is a positive constant and $0<\beta \leq 1$. It turns out that $s(t)$ is Lipschitz for $0<t<\infty$ and Hölder continuous on $[0, \infty)$ with exponent $\gamma=(1+\beta)^{-1}$, and that $\hat{u}_{x}$ and $\hat{u}_{t}$ are Hölder continuous with exponent $\gamma$ everywhere except at the origin.

The main tool used below is the boundary point form of the maximum principle: 
if $v$ is continuous and nonnegative in the closure of a domain $D$ which is bounded below by $t=0$ and on the right by a Lipschitz curve $x=s(t)$, and if $L v \leq 0$ in $D$ and $v\left(s\left(t_{0}\right), t_{0}\right)=0$ for some $t_{0}>0$, then $v_{x}\left(s\left(t_{0}\right), t_{0}\right)<0$ (cf., e.g., A. Friedman, Remarks on the maximum principle for parabolic equations and its applications, Pacific J. Math. 8 (1958), 201-211). In particular, the optimality theorems mentioned above follow from an application of this result. We remark here that, although the usual statements of this result require that $D$ satisfy an "interior sphere condition" at $\left(s\left(t_{0}\right), t_{0}\right)$, it is a simple matter to achieve this by modifying the situation for $t>t_{0}$ without altering $v$ for $t \leq t_{0}$ as long as $s(t)$ is Lipschitz.

2. Properties of the solution. Let $x=s(t)$ be a curve defined for $0 \leq t<\infty$ with $s(t)>0$, and let $u$ be a real-valued function defined on the open set

$$
C=\{(x, t) ; 0<x<s(t), 0<t<\infty\} \text {. }
$$

Definition 1. $\{s, u\}$ is a solution of (1.2)-(1.7) if

(i) $s \in C^{\gamma}([0, \infty))$ for some $\gamma \in[1 / 2,1)$ and is Lipschitz continuous for $0<$ $t<\infty$.

(ii) $u \in C^{2,1}(C) \cap C^{0}(\bar{C})$.

(iii) $\partial u / \partial x$ is bounded and has a continuous extension to $\bar{C}$.

(iv) $\partial^{2} u / \partial x^{2}$ is bounded and has a continuous extension to $\bar{C}-(0,0)-$ $(s(0), 0)$.

(v) Conditions (1.2)-(1.7) are satisfied.

We begin with a number of simple properties which solutions must satisfy.

Lemma 1. Let $\{s, u\}$ be a solution of (1.2)-(1.7). Then $s(0)=d$ and $s(t) \geq$ $d$ for $0<t<\infty$.

Proof. First suppose $s(0)>d$, and choose $x_{0} \in(d, s(0))$. Then

$$
\lim _{t \rightarrow 0} \frac{\partial u}{\partial t}\left(x_{0}, t\right)=\lim _{t \rightarrow 0}\left(L_{0} u\right)\left(x_{0}, t\right)=L_{0} \psi\left(x_{0}\right)<0,
$$

which contradicts (1.3). Hence, $s(0) \leq d$.

Now suppose there exists $t_{0}$ such that $s\left(t_{0}\right)<d$. Then there exist $t_{1}, t_{2}$ such that $0 \leq t_{1}<t_{2}$ and $s(t)<d\left(t_{1} \leq t \leq t_{2}\right)$. Let $D=\left\{(x, t) ; 0<x<s(t), t_{1}<\right.$ $\left.t<t_{2}\right\}$, and define $v=u-\psi$ on $\bar{D}$. Then $v \geq 0$ on $\bar{D}$ by (1.3) and $L v \leq 0$ by (1.1) and (1.2). Moreover, $v(s(t), t)=0 \quad\left(t_{1} \leq t \leq t_{2}\right)$ by (1.6). But then we must have $v_{x}(s(t), t)<0\left(t_{1}<t \leq t_{2}\right)$ by the boundary point form of the maximum principle and this contradicts (1.7). Hence, $s(t) \geq d$ for all $t \geq 0$, which proves the lemma.

Lemma 2. Let $\{s, u\}$ be a solution of (1.2)-(1.7). Then $u_{t}>0$ in $C$, and $s(t)$ is nondecreasing. 
Proof. First observe that (1.6) implies that

$$
u_{x}(s(t), t) s^{\prime}(t)+u_{t}(s(t), t)=\psi^{\prime}(s(t)) s^{\prime}(t)
$$

for each $t>0$ where $s(t)$ is differentiable. By (1.7), this implies $u_{t}(s(t), t)=0$ for almost every $t>0$. But then $u_{t}(s(t), t) \equiv 0$ by (iv) of Definition 1 . We also have $u_{t}(0, t)=0(t>0)$ and $u_{t}(x, 0) \geq 0(0<x<d)$ by (1.4) and (1.1) respectively. Furthermore, the derivatives $u_{x x t}, u_{x t}, u_{t t}$ exist and are (Hölder) continuous in $C$ by an interior regularity theorem of Friedman [6], and hence $L u_{t} \equiv 0$ in $C$. It follows that $u_{t}>0$ in $C$ by the strong maximum principle.

If $s(t)$ is not nondecreasing, we can find $t_{0}$ such that $s^{\prime}\left(t_{0}\right)$ exists and is $<0$. Then $\left(s\left(t_{0}\right), t_{0}-\delta\right) \in C$ for all sufficiently small positive $\delta$. But then

$$
\psi\left(s\left(t_{0}\right)\right)-u\left(s\left(t_{0}\right), t_{0}-\delta\right)=\delta u_{t}\left(s\left(t_{0}\right), t_{0}-\delta^{\prime}\right)>0
$$

for some $\delta^{\prime} \epsilon(0, \delta)$, and this contradicts (1.3). This completes the proof.

Remark. In view of the monotonicity of $s(t)$, the proof of Lemma 1 actually shows that $s(t)>d(t>0)$.

We now characterize the solution as the solution of a minimum problem concerning supersolutions relative to the operator $L$.

Notation.

$$
\begin{aligned}
R_{\delta}\left(x_{0}, t_{0}\right) & =\left\{(x, t) ;\left|x-x_{0}\right|<\delta, t_{0}-\delta<t \leq t_{0}\right\}, \\
\partial_{p} R_{\delta}\left(x_{0}, t_{0}\right) & =\left\{(x, t) \in \partial R_{\delta}\left(x_{0}, t_{0}\right) ; t \neq t_{0}\right\} .
\end{aligned}
$$

Definition 2. A function $v=v(x, t)$ is an L-supersolution if

(i) $v$ is defined and lower semicontinuous on $\bar{Q}$.

(ii) For any $\left(x_{0}, t_{0}\right) \in Q$, there exists $\delta_{0}>0$ such that $R_{\delta_{0}}\left(x_{0}, t_{0}\right) \subseteq \bar{Q}$ and for all $\delta \in\left(0, \delta_{0}\right)$ we have that $v\left(x_{0}, t_{0}\right) \geq w\left(x_{0}, t_{0}\right)$ whenever $w \in C^{2}, 1\left(R_{\delta}\right)$ $\cap C^{0}\left(\bar{R}_{\delta}\right), L w=0$ in $R_{\delta}$, and $v \geq w$ on $\partial_{p} R_{\delta}$.

Theorem 1. Let $\{s, u\}$ be a solution of (1.2)-(1.7), and let $\hat{u}$ be defined on $\bar{Q}$ by (1.8). Then $\hat{u}$ is the least L-supersolution on $\bar{Q}$ which is $\geq \psi$ on $\bar{Q}$.

Proof. We have $\hat{u} \geq \psi$ by (1.3), and $\hat{u}$ is continuous by (1.6). We show that $\hat{u}$ is an $L$-supersolution. If $\left(x_{0}, t_{0}\right)$ is in either the continuation region or the interior of its complement, condition (ii) of Definition 2 is verified for $\delta_{0}$ equal to the distance of $\left(x_{0}, t_{0}\right)$ from $\partial C \cup\{t=0\}$ by application of the maximum principle. If $x_{0}=s\left(t_{0}\right), t_{0}>0$, we choose $\delta_{0}>0$ so small that the closure of $R_{\delta_{0}}\left(x_{0}, t_{0}\right)$ is contained in the open quarter plane $x>d, t>0$. Define

$$
D_{1, \delta}=R_{\delta}\left(x_{0}, t_{0}\right) \cap\{s(t)>x\}, \quad D_{2, \delta}=R_{\delta}\left(x_{0}, t_{0}\right) \cap\{s(t)<x\}
$$


for $\delta \in\left(0, \delta_{0}\right)$. Let $w$ be as in condition (ii) of Definition 2, and set $v=u-w$, $m=\inf \bar{R} \delta$. Suppose $m<0$. By the maximum principle, $v(x, t) \neq m$ for $(x, t) \epsilon$ $D_{1, \delta} \cup D_{2, \delta} \cup \partial_{p} R_{\delta}$. Therefore, there exists a point $\left(s\left(t_{1}\right), t_{1}\right), t_{0}-\delta<t_{1} \leq t_{0}$. such that $v\left(s\left(t_{1}\right), t_{1}\right)=m$. By the boundary point form of the maximum principle,

$$
v_{x}\left(s\left(t_{1}\right)-, t_{1}\right)<0, \quad v_{x}\left(s\left(t_{1}\right)+, t_{1}\right)>0 .
$$

But this contradicts (1.7) and the differentiability of $w$. Hence $m \geq 0$, which verifies condition (ii) of Definition 2.

Now let $w$ be another $L$-supersolution such that $w \geq \psi$ in $\bar{Q}$. Set $v=w-u$ and, for any $T>0$, let $m_{T}=\inf _{\bar{Q}_{T}} v$, where $Q_{T}=[0, \infty) \times(0, T]$. Assume $m_{T}<$ 0 . Since $v \geq 0$ outside $C$ and $v$ is lower semicontinuous, the set $S_{T}=\{(x, t) \epsilon$ $\left.\bar{Q}_{T} ; v(x, t)=m_{T}\right\}$ is a nonvoid closed set lying in $C$. Hence, there exists a point $\left(x_{0}, t_{0}\right) \in S_{T}$ such that $t_{0}>0$ and $(x, t) \notin S_{T}$ if $t<t_{0}$. If $\delta>0$ is sufficiently small, we have $R_{\delta}\left(x_{0}, t_{0}\right) \subseteq C$. Let $\tilde{u}$ be the solution of the first initial-boundary value problem

$$
L \tilde{u}=0 \quad \text { in } R_{\delta}\left(x_{0}, t_{0}\right), \quad \tilde{u}=u+m_{T} \text { on } \lambda_{p} R_{\delta}\left(x_{0}, t_{0}\right) .
$$

Since $\partial_{p} R_{\delta} \subseteq C-S_{T}$, we have $w>\widetilde{u}$ on $\partial_{p} R_{\delta}$, and hence $w\left(x_{0}, t_{0}\right) \geq \tilde{u}\left(x_{0}, t_{0}\right)$ if $\delta$ is sufficiently small since $w$ is an $L$-supersolution. But $\tilde{u}>u+m_{T}$ in $R_{\delta}$ by the strong maximum principle since

$$
L\left(\tilde{u}-u-m_{T}\right)=-c(x) m_{T} \leq 0 \quad \text { in } R_{\delta}, \quad \tilde{u}-u-m_{T}=0 \text { on } \partial_{p} R_{\delta} .
$$

Therefore, $v\left(x_{0}, t_{0}\right)>m_{T}$, which contradicts our choice of the point $\left(x_{0}, t_{0}\right)$. Hence, $m_{T} \geq 0$ and $w \geq \hat{u}$ in $\bar{Q}_{T}$. Therefore, $w \geq \hat{u}$ in $\bar{Q}$.

Theorem 2 (Uniqueness), Let $\left\{s_{1}, u_{1}\right\}$ and $\left\{s_{2}, u_{2}\right\}$ be two solutions of (1.2)-(1.7). Then $s_{1} \equiv s_{2}$ and $u_{1} \equiv u_{2}$.

Proof. Let $C_{1}, C_{2}$ be the continuation regions defined by $s_{1}, s_{2}$ respectively, and let $\hat{u}_{i}(i=1,2)$ be defined as in (1.8). Then $u_{1} \equiv u_{2}$ by Theorem 1. Suppose $s_{1}(t)<s_{2}(t)$ for some $t$. Then there is an open set $D \subseteq C_{2} \cap\left(Q-C_{1}\right)$ in which we have

$$
0=L u_{2}=L \hat{u}_{2}=L \hat{u}_{1}=L \psi
$$

But this contradicts (1.1). Hence, $s_{1} \equiv s_{2}$ and $u_{1} \equiv u_{2}$.

We now establish the connection between the free boundary problem and the optimal stopping problem, which was described in $\$ 1$.

Theorem 3. Let $\{s, u\}$ be a solution of (1.2)-(1.7), and let $\hat{u}$ be defined as 
in (1.8). Let

$$
v(x, t)=\sup _{\bar{m} \in \bar{M}} E_{X(-t)=x}(\psi(X(\bar{m})))
$$

and

$$
\bar{m}_{\mathrm{opt}}=\inf \{t ;(X(t),-t) \notin C\}
$$

Then

$$
\hat{u}(x, t)=v(x, t)=E_{X(-t)=x}\left(\psi\left(X\left(\bar{m}_{\mathrm{opt}}\right)\right)\right)
$$

for all $(x, t) \in \bar{Q}$.

Proof. Since $\hat{u}(x, t)=E_{X(-t)=x}\left(\psi\left(X\left(\bar{m}_{\text {opt }}\right)\right)\right)$, we certainly have $u \leq v$ on $\bar{Q}$. The problem is to prove the reverse inequality.

If $\phi \in C_{0}^{\infty}(Q)$ and $L^{*}$ is the formal adjoint of $L$, then

$$
\begin{aligned}
\int_{Q} \hat{u} L^{*} \phi & =\int_{C} u L^{*} \phi+\int_{Q-C} \psi L^{*} \phi \\
& =\int_{C}(L u) \phi+\int_{Q-C}(L \psi) \phi=\int_{Q}\left(1-\chi_{C}\right)(L \psi) \phi
\end{aligned}
$$

where $\chi_{C}$ is the characteristic function of $C$, for the boundary terms generated by the integrations by parts cancel by virtue of (1.6) and (1.7). Hence, $L \hat{u}=$ $\left(1-\chi_{C}\right) L \psi$ in $Q$ in the distribution sense. By Duhamel's principle, $\hat{u}$ may then be represented in the form

$$
\hat{u}(x, t)=u_{1}(x, t)+\int_{0}^{t} w_{\tau}(x, t) d \tau
$$

where $u_{1}$ is the solution of the problem $L u_{1}=0$ in $Q, u_{1}=\psi$ on $\partial Q$ and $w_{\tau}(x, t)$ is defined for $r \leq t<\infty$ as the solution of the problem

$$
\begin{aligned}
L w_{\tau} & =0 \quad(x>0, t>\tau), \\
w_{\tau}(0, t) & =0 \quad(t>\tau), \\
w_{\tau}(x, \tau) & =\left(1-\chi_{C}(x, \tau)\right)(L \psi)(x, \tau) .
\end{aligned}
$$

Now consider the Markov process with state space $\bar{Q}$ whose sample paths starting at $(x, t)$ are the backwards graphs $r \rightarrow(X(r-t), t-r)(0 \leq r \leq t)$ of the sample paths of the original process with starting condition $X(-t)=x$. The generator of this (space-time) process agrees on smooth functions with the differential operator $L$. The induced semigroup of operators on Borel functions defined on $\vec{Q}$ is given by 


$$
\begin{aligned}
H_{\tau} f(x, t) & =E_{x} f(X(\tau), t-\tau) & & \text { if } 0 \leq \tau<t, \\
& =0 & & \text { if } \tau \geq t,
\end{aligned}
$$

and the corresponding Green operators are

$$
G_{a} f(x, t)=\int_{0}^{t} e^{-a \tau_{\tau} f(x, t) d \tau \quad(\alpha \geq 0) .}
$$

Set $f=\left(1-\chi_{C}\right)(L \psi)$. Then $E_{x} f(X(\tau), t-\tau)=w_{\tau}(x, t)$, so that

$$
G_{0} f(x, t)=\int_{0}^{t} w_{\tau}(x, t) d \tau=\hat{u}(x, t)-u_{1}(x, t) \equiv \tilde{u}(x, t) .
$$

Hence, by Theorem 1 on p. 67 of [10], for all $\bar{m} \in \bar{M}$ we have

$$
\begin{aligned}
\tilde{u}(x, t) & =E_{X(-t)=x}\left(\int_{-t}^{\bar{m}} f(X(\tau),-\tau) d \tau\right)+E_{X(-t)=x}(\tilde{u}(X(\bar{m}),-\bar{m})) \\
& \geq E_{X(-t)=x}(\tilde{u}(X(\bar{m}),-\bar{m}))
\end{aligned}
$$

since $f \geq 0$. Since $u_{1}$ is smooth and $L u_{1}=0$, we have

$$
u_{1}(x, t)=E_{X(-t)=x}\left(u_{1}(X(\bar{m}),-\bar{m})\right)
$$

for all $\bar{m} \in \bar{M}$ by Theorem 9 on p. 85 of [10]. Therefore,

$$
\hat{u}(x, t) \geq E_{X(-t)=x}(\hat{u}(X(\bar{m}),-\bar{m})) \geq E_{X(-t)=x}(\psi(X(\bar{m})))
$$

by (1.3). Taking the supremum over all $\bar{m} \in \bar{M}$, we arrive at the desired inequality.

We now tum to the steady state analogue of the free boundary problem (1.2)(1.7). By comparing the steady and unsteady cases, we obtain a priori bounds on the solution of (1.2)-(1.7) and its limiting behavior as $t \rightarrow \infty$.

Theorem 4. Let $u_{s}$ denote the solution of the boundary value problem

$$
L_{0} u_{s}=0 \quad(0<x<s), \quad u_{s}(0)=\psi(0), \quad u_{s}(s)=\psi(s) .
$$

Assume that

$$
\underset{\xi \rightarrow \infty}{\lim \sup _{0}}\left[L_{0} \psi\right](\xi)<0
$$

Then there exists a unique value $s=s_{\infty}$ such that

$$
u_{s}^{\prime}(s)=\psi^{\prime}(s) \text {. }
$$

Moreover, $s_{\infty}>d$ and $u_{\infty} \equiv u_{s_{\infty}}>\psi\left(0<x<s_{\infty}\right)$. 
Proof. We remark that the solution $u_{s}$ of (2.1) always exists since $c(x) \leq 0$. Let $L_{0}^{*}$ denote the formal adjoint of $L_{0}$, and let $W$ be the solution of the initial value problem $L_{0}^{*} W=0, W(0)=0, W^{\prime}(0)=1$. Since $W$ cannot have a positive maximum, we have

$$
W(\xi)>0 \quad(0<\xi<\infty), \quad \underset{\xi \rightarrow \infty}{\lim \inf } W(\xi)>0 .
$$

Apply the Green identity

$$
\int_{0}^{s}\left[\left(L_{0} v\right) w-v\left(L_{0}^{*} w\right)\right] d \xi=\left[d(\xi)\left(v^{\prime}(\xi) w(\xi)-v(\xi) w^{\prime}(\xi)\right)+b(\xi) v(\xi) w(\xi)\right]_{\xi=0}^{\xi=s}
$$

with $v=\psi-u_{s}, w=W$; the result is

$$
a(s) W(s)\left[\psi^{\prime}(s)-u_{s}^{\prime}(s)\right]=\int_{0}^{s}\left[L_{0} \psi\right](\xi) W(\xi) d \xi .
$$

In view of (1.1), (2.2) and (2.4), there is a unique positive value of $s=s_{\infty}$ such that the right-hand side of (2.5) vanishes. This proves the existence of a unique solution $\left\{s_{\infty}, u_{\infty}\right\}$ of (2.1), (2.3). That $s_{\infty}>d$ is clear.

Since $u_{\infty}\left(s_{\infty}\right)=\psi\left(s_{\infty}\right)$ and $u_{\infty}^{\prime}\left(s_{\infty}\right)=\psi^{\prime}\left(s_{\infty}\right)$, it follows that

$$
0>\left[L_{0}\left(\psi-u_{\infty}\right)\right]\left(s_{\infty}\right)=a\left(s_{\infty}\right)\left[\psi^{\prime \prime}\left(s_{\infty}\right)-u_{\infty}^{\prime \prime}\left(s_{\infty}\right)\right] .
$$

Hence, $u_{\infty}(x)>\psi(x)$ in some interval $s_{\infty}-\delta<x<s_{\infty}(\delta>0)$. Let $s_{0}$ be the largest value of $x<s_{\infty}$ such that $u_{\infty}(x)=\psi(x)$. Then $0 \leq s_{0}<d$, for otherwise the facts that $L_{0}\left(\psi-u_{\infty}\right)<0\left(s_{0}<x<s_{\infty}\right)$ and $\psi(x)=u(x)$ for $x=s_{0}$, $s_{\infty}$ would imply that $\psi>u_{\infty}$ for $s_{0}<x<s_{\infty}$ by the maximum principle. Likewise, if $0<$ $s_{0}<d$, the maximum principle implies that $u_{\infty}(x) \geq \psi(x)$ for $0<x<s_{0}$, so that $u_{\infty}-\psi$ has an interior minimum at $x=s_{0}$. Consequently, $u_{\infty}^{\prime}\left(s_{0}\right)=\psi^{\prime}\left(s_{0}\right)$. But this is impossible by (2.5) since $u_{\infty} \equiv u_{s_{0}}$ if $u_{\infty}\left(s_{0}\right)=\psi\left(s_{0}\right)$. Hence, $s_{0}=0$, which proves that $u_{\infty}>\psi$ in $0<x<s_{\infty}$.

Theorem 5. Let $\{s, u\}$ be a solution of (1.2)-(1.7), and let $\left\{s_{\infty} u_{\infty}\right\}$ be the solution of (2.1), (2.3). Then

$$
s(t)<s_{\infty} \quad(0 \leq t<\infty), \quad u(x, t)<u_{\infty} \quad \text { in } C .
$$

Moreover,

$$
s(t) \rightarrow s_{\infty}, \quad u(x, t) \rightarrow u_{\infty}(x) \text { uniformly as } t \rightarrow \infty .
$$

Proof. It is easy to see that the function

$$
\hat{u}_{\infty}(x)= \begin{cases}u_{\infty}(x), & 0 \leq x \leq s_{\infty}, \\ \psi(x), & s_{\infty}<x<\infty,\end{cases}
$$


is an $L$-supersolution on $\bar{Q}$. Hence, $\hat{u}_{\infty} \geq u$ on $C$. Moreover, $s(t)<s_{\infty}($ all $t \geq 0)$ implies $u_{\infty}>u$ on $C$ by the strong maximum principle. Assume that (2.6) fails. Then there exists a smallest time $t_{0}>0$ such that $s\left(t_{0}\right)=s_{\infty}$. Then $L\left(u_{\infty}-u\right)=$ 0 in $0<x<s(t), 0<t \leq t_{0}$ and $\left(u_{\infty}-u\right)$ has a nonpositive minimum at $\left(s_{\infty} t_{0}\right)$. By the boundary point maximum principle, it follows that $\left(u_{\infty}-u\right)_{x}<0$ at $\left(s_{\infty}, t_{0}\right)$, which contradicts (1.7) and (2.3). Thus, (2.6) is proved.

Now we apply Green's formula

$$
\iint_{C \cap Q_{\ell}}\left[(L v) w-v\left(L^{*} w\right)\right] d x \wedge d t=\oint_{\partial\left(C \cap Q_{t}\right)}\left\{\left[a\left(v_{x} w-v w_{x}\right)+b v w\right] d t+v w d x\right\}
$$

with $v=\psi-u$ and $w=W(W$ as in the proof of Theorem 4). The result is

$$
\int_{0}^{t}\left[\int_{0}^{s(\tau)}\left(L_{0} \psi\right)(\xi) W(\xi) d \xi\right] d \tau+\int_{0}^{s(\ell)}[\psi(\xi)-u(\xi, t)] W(\xi) d \xi=0
$$

Since $s(t)$ and $u(x, t)$ are bounded above by (2.6) and nondecreasing in $t$ by Lemma 1 , the limits

$$
s^{\infty}=\lim _{t \rightarrow \infty} s(t), \quad u^{\infty}(x)=\lim _{t \rightarrow \infty} u(x, t)
$$

exist. Moreover, the second term in (2.8) is bounded. Since $s(r)>d$ is nondecreasing, the bracketed integral in the first term of $(2.8)$ is nonincreasing. Since the first term must be bounded, this implies that

$$
\lim _{t \rightarrow \infty} \int_{0}^{s(t)}\left[L_{0} \psi\right](\xi) W(\xi) d \xi=\int_{0}^{s^{\infty}}\left[L_{0} \psi\right](\xi) W(\xi) d \xi=0
$$

Therefore, $s^{\infty}=s_{\infty}$.

Now let $\hat{u}(x, t)$ be defined by (1.8), and let $U$ be the solution of $L U=0$ in the half-strip $0<x<s_{\infty}, t>0$ which equals $\psi$ on the boundary. Since $\hat{u}$ is an $L$-supersolution, it may be shown that $\hat{u} \geq U$ in the half-strip (cf. the last paragraph of the proof of Theorem 1). But $u_{\infty} \geq \hat{u}$, and $U(x, t) \rightarrow u_{\infty}(x)$ uniformly in $x$ by elementary methods, so that $\lim _{t \rightarrow \infty} \hat{u}(x, t)=u^{\infty}(x)=u_{\infty}(x)$, and the convergence is uniform.

3. Construction of the solution. We now construct a discrete-time analogue of problem (1.2)-(1.7) and prove that its solution converges to a solution of of (1.2)-(1.7) as the time step tends to zero. The possibility of solving the approximate problem rests on the following lemma. 
Lemma 3. Let $\lambda>0$ be given, and let

$$
M=\sup _{0 \leq x \leq s_{\infty}}|\psi(x)| .
$$

Assume that $s \in\left[d, s_{\infty}\right)$ and $v \in C^{2}([0, s])$ are given sucb that $v \geq \psi,|v| \leq M$, $L v \geq 0$ but $\neq 0$ on $[0, s], v(0)=0, v(s)=\psi(s), v^{\prime}(s)=\psi^{\prime}(s)$. Let $\hat{v}$ be defined on $[0, \infty)$ by

$$
\hat{v}(x)= \begin{cases}v(x), & 0 \leq x \leq s \\ \psi(x), & s<x<\infty\end{cases}
$$

Assume that (2.2) bolds.

Then there exists a unique number $s^{\prime}>s$ sucb that the solution $u_{\sigma}$ of the problem

$$
\begin{aligned}
& L_{0} u_{\sigma}=\lambda\left(u_{\sigma}-\hat{v}\right) \quad \text { on }(0, \sigma), \\
& u_{\sigma}(0)=\psi(0), \quad u_{\sigma}(\sigma)=\psi(\sigma)
\end{aligned}
$$

also satisfies

$$
u_{\sigma}^{\prime}(\sigma)=\psi^{\prime}(\sigma)
$$

exactly when $\sigma=s^{\prime}$. Moreover, $s^{\prime}<s_{\infty}$ and we have $|u| \leq M, u \geq \hat{v}$ and $L_{0} u \geq$ 0 but not $\equiv 0$ on $\left[0, s^{\prime}\right]$ where $u=u_{s^{\prime}}$.

Proof. Let $W$ be the solution of the initial value problem

$$
L_{0}^{*} W-\lambda W=0, \quad W(0)=0, \quad W^{\prime}(0)=1
$$

Since $W$ cannot have a positive maximum, it follows that

$$
W(\xi)>0 \quad(\xi>0), \quad \lim _{\xi \rightarrow \infty} \inf W(\xi)>0 .
$$

By (3.1) and (3.3), we have

$$
\int_{0}^{\sigma}\left[\left(L_{0} u_{\sigma}\right) W-u_{\sigma}\left(L_{0}^{*} W\right)\right] d \xi=-\int_{0}^{\sigma} \hat{v}(\xi)\left[L_{0}^{*} W\right](\xi) d \xi .
$$

By applying Green's identity to both sides and substituting the boundary values of $u_{\sigma}$ and $\hat{v}$, we arrive at the equation

(3.5) $a(\sigma) W(\sigma)\left[\psi^{\prime}(\sigma)-u_{\sigma}^{\prime}(\sigma)\right]=\int_{0_{0}}^{s}\left[L_{0} \nu\right](\xi) W(\xi) d \xi+\int_{s}^{\sigma}\left[L_{0} \psi\right](\xi) W(\xi) d \xi$.

In view of (1.1), (2.2), (3.4) and the assumption about $L_{0} v$ on $[0, s]$, the right- 
hand side of (3.5) has a unique zero $\sigma=s^{\prime}>s$. There follows the unique solvability of (3.1), (3.2).

Since $u\left(s^{\prime}\right)=\psi\left(s^{\prime}\right), u^{\prime}\left(s^{\prime}\right)=\psi^{\prime}\left(s^{\prime}\right)$, we have

$$
a\left(s^{\prime}\right)\left[u^{\prime \prime}\left(s^{\prime}\right)-\hat{v}^{\prime \prime}\left(s^{\prime}\right)\right]=\left[L_{0}(u-\hat{v})\right]\left(s^{\prime}\right)=-\left[L_{0} \psi\right]\left(s^{\prime}\right)>0 .
$$

Hence, $u>\hat{v}$ in some neighborhood $s^{\prime}-\delta<x<s^{\prime}(\delta>0)$. Suppose that the inequality $u>\hat{v}$ fails in the interval $\left[s, s^{\prime}\right)$. Let $s_{0}=\sup \left(x \in\left[s, s^{\prime}\right) ; u(x)=\right.$ $\hat{v}(x)\}$. Then $u^{\prime}\left(s_{0}\right) \geq \hat{v}^{\prime}\left(s_{0}\right)=\psi^{\prime}\left(s_{0}\right)$. Since $u_{s_{0}} \equiv u$ on $\left[0, s_{0}\right]$, it follows that the right side of (3.5) is nonpositive for $\sigma=s_{0}<s^{\prime}$. But this is impossible. Therefore, $u(x)>\hat{v}(x)\left(s \leq x<s^{\prime}\right)$.

Now let $w=u-v$ on $[0, s]$. Then $w$ satisfies

$$
\left(L_{0}-\lambda\right) w=-L_{0} v \leq 0, \quad w(0)=0, \quad w(s)=u(s)-\psi(s)>0 .
$$

Hence, $w \geq 0$ on $[0, s]$ by the maximum principle, and we have shown that $u \geq \hat{v}$ on $\left[0, s^{\prime}\right]$. By (3.1), we also have $L_{0} u \geq 0$ in $\left[0, s^{\prime}\right]$. We have already seen that $L_{0} u\left(s^{\prime}\right)>0$, so $L_{0^{u}} \not 0$.

Next, suppose that $s^{\prime} \geq s_{\infty}$. Let $w_{1}$ be the solution of (3.1) with $\sigma=s_{\infty}$. Then $\psi^{\prime}\left(s_{\infty}\right) \geq w_{1}^{\prime}\left(s_{\infty}\right)$ by (3.5) with $\sigma=s_{\infty}$. This implies that $w_{1} \geq \hat{v}$ on $\left[0, s_{\infty}\right]$ by the same reasoning given above for $u$. Therefore, $L_{0}\left(u_{\infty}-w_{1}\right)=$ $-\lambda\left(w_{1}-\hat{v}\right) \leq 0$. But then $u_{\infty}-w_{1}$ must take its maximum value on $\left[0, s_{\infty}\right]$ at $x=s_{\infty}$, and furthermore $u_{\infty}^{\prime}\left(s_{\infty}\right)=\psi^{\prime}\left(s_{\infty}\right)<w_{1}^{\prime}\left(s_{\infty}\right)$. But this is a contradiction. Hence, $s^{\prime}<s_{\infty}$.

Finally, on $\left[0, s^{\prime}\right]$ we clearly have

$$
M \geq \max \left[\psi(0), \psi\left(s_{\infty}\right)\right] \geq u_{\infty} \geq u \geq \hat{v} \geq \psi \geq-M
$$

so that $|u| \leq M$ there.

We now construct the discrete time approximation to problem (1.2)-(1.7).

Let $b>0$ be a time step and set $s_{0}=d, u_{0}(x)=\psi(x)(0 \leq x \leq d)$. Then define sequences $\left\{s_{n}\right\},\left\{u_{n}(x)\right\}$ inductively by solving at each stage the problem

$$
\begin{aligned}
& \left(u_{n}(x)-\hat{u}_{n-1}(x)\right) / b=\left[L_{0} u_{n}\right](x) \quad\left(0<x<s_{n}\right) \\
& u_{n}(0)=\psi(0), \quad u_{n}\left(s_{n}\right)=\psi\left(s_{n}\right), \quad u_{n}^{\prime}\left(s_{n}\right)=\psi^{\prime}\left(s_{n}\right)
\end{aligned}
$$

for $s_{n}$ and $u_{n}(x)$. Here

$$
\hat{u}_{n}(x)= \begin{cases}u_{n}(x), & 0 \leq x \leq s_{n}, \\ \psi(x), & s_{n}<x<\infty .\end{cases}
$$

This construction is possible by virtue of Lemma 3. Moreover, Lemma 3 guarantees that 
(i) $d=s_{0}<s_{1}<\cdots<s_{n}<\cdots<s_{\infty}$,

(ii) $\hat{u}_{n}(x) \leq u_{n+1}(x) \quad\left(0 \leq s \leq s_{n+1}\right), n=0,1,2, \cdots$,

(iii) $\left|u_{n}(x)\right| \leq M$ and $\left[L_{0} u_{n}\right](x) \geq 0 \quad\left(0 \leq x \leq s_{n} ; n=0,1,2, \ldots\right)$.

As the analysis of this scheme is rather lengthy, we break it up into a progression of lemmas.

Lemma 4. Let $\hat{u}_{-1}(x)=\psi(x)-b\left[L_{0} \psi\right](x)$ and $s_{-1}=d$. Define

$$
q_{n}(x)=\left(u_{n}(x)-\hat{u}_{n-1}(x)\right) / b \quad\left(0 \leq x \leq s_{n}\right)
$$

for $n=0,1,2, \ldots$. Then there exist constants $M_{0}$ and $M_{1}$, which depend only on $\psi$ and on the coefficients of $L$, such that $0 \leq q_{n}(x) \leq M_{0}(n=0,1,2, \ldots)$, $\left|u_{n}^{\prime}(x)\right| \leq M_{1}(n=0,1,2, \ldots)$.

Proof. Note that $q_{n}(x)$ satisfies the equations

$$
\begin{aligned}
L_{0} q_{n}(x) & =\left\{\begin{array}{ll}
{\left[q_{n}(x)-q_{n-1}(x)\right] / b} & \text { if } 0 \leq x \leq s_{n-1} \\
{\left[q_{n}(x)-L_{0} \psi(x)\right] / b} & \text { if } s_{n-1}<x \leq s_{n}
\end{array} \quad(n=1,2, \ldots)\right. \\
q_{0}(x) & =L_{0} \psi(x),
\end{aligned}
$$

and that $q_{n}(0)=q_{n}\left(s_{n}\right)=q_{n}^{\prime}\left(s_{n}\right)=0$ and $L_{0} q_{n}\left(s_{n}\right)>0$ for $n=1,2, \ldots$ Hence, $q_{n}^{\prime \prime}\left(s_{n}\right)>0$, from which it follows that $q_{n}(x)>0$ in some neighborhood $s_{n}-\delta<$ $x<s_{n}$. Therefore, $q_{n}(x)$ has a positive interior maximum at some point $x_{0} \epsilon$ $\left(0, s_{n}\right)$. But we cannot have $s_{n-1}<x_{0}<s_{n}$ since then

$$
0 \geq L_{0} q_{n}\left(x_{0}\right)=b^{-1}\left[L_{0} u_{n}\left(x_{0}\right)-L_{0} \psi\left(x_{0}\right)\right]>0
$$

by (3.7). Hence, $0 \leq x_{0} \leq s_{n-1}$, whence $q_{n}\left(x_{0}\right) \leq q_{n-1}\left(x_{0}\right)$ by (3.7). Therefore,

$$
0 \leq q_{n}(x) \leq \max _{0 \leq \xi \leq d} L_{0} \psi(\xi) \equiv M_{0} \quad(n=0,1,2, \cdots) .
$$

Next, observe that

$$
\begin{aligned}
a(x) u_{n}^{\prime}(x)= & -\int_{x}^{s_{n}} L_{0} u_{n}(\xi) d \xi+d\left(s_{n}\right) \psi^{\prime}\left(s_{n}\right)+b\left(s_{n}\right) \psi\left(s_{n}\right)-b(x) u_{n}(x) \\
& +\int_{x}^{s}{ }^{n} u_{n}(\xi) c(\xi) d \xi .
\end{aligned}
$$

Therefore, $\left|u_{n}^{\prime}(x)\right| \leq a_{0}^{-1}\left[A \max _{\left[0, s_{\infty}\right]}\left|\psi^{\prime}\right|+\left(2 B+s_{\infty} C\right) M+s_{\infty} M_{0}\right] \equiv M_{1}$, where $A, B, C$ are the maxima of $|a|,|b|,|c|$ respectively on $\left[0, s_{\infty}\right]$.

The next lemma provides us with an inequality which guarantees the uniform Hölder continuity of the approximating free boundary curves and forces 
their convergence. This aspect of the existence proof may be regarded as the most novel technical feature of the problem under consideration.

Lemma 5. Define, for $d \leq x<\infty$, a nonnegative strictly increasing function $G(x)$ by

$$
G(x)=-\int_{d}^{x}\left(L_{0} \psi\right)(\xi) d \xi
$$

Then, for each $T>0$, there exists a constant $M_{2}(T)$, depending only on $\psi$ and the coefficients of $L$, such that

$$
0<\left[G\left(s_{n}\right)-G\left(s_{n-1}\right)\right] / b \leq M_{2}(T) \quad(n=1,2, \cdots,[T / b]) .
$$

Proof. For $n=0,1,2, \ldots$, we introduce the functions

$$
p_{n}(x)=a(x) q_{n}^{\prime}(x)+b(x) q_{n}(x) .
$$

Since $q_{n}(x)$ is continuously differentiable on $\left[0, s_{n}\right]$, Green's formula yields

$$
p_{n}(x)=-\int_{x}^{s}\left(L_{0} q_{n}\right)(\xi) d \xi+\int_{x}^{s} n d(\xi) q_{n}(\xi) d \xi
$$

By (3.7), it follows that

$$
p_{n}\left(s_{n-1}\right)=-\int_{s_{n-1}}^{s_{n}} \frac{q_{n}(\xi)-L_{0} \psi(\xi)}{b} d \xi+\int_{s_{n-1}}^{s_{n}} c(\xi) q_{n}(\xi) d \xi
$$

so that

$$
p_{n}\left(s_{n-1}\right) \leq-\left(G\left(s_{n}\right)-G\left(s_{n-1}\right)\right) / b
$$

The lemma will then be established if we can obtain a lower bound $p_{n}(x) \geq-$ $-M_{2}(T)$ (we remark that we cannot expect a similar upper bound since appropriate compatibility conditions at the origin are not satisfied).

Since (for $n \geq 1$ ) we have $p_{n}\left(s_{n}\right)=0$ and

$$
p_{n}^{\prime}(x)=\left(q_{n}(x)-L_{0} \psi(x)\right) / b-c(x) q_{n}(x)>0 \quad\left(s_{n-1}<x \leq s_{n}\right)
$$

by (3.7) and (3.8), it follows that $p_{n}(x)<0\left(s_{n-1}<x<s_{n}\right) . p_{n}(x)$ cannot have its minimum value in $\left(s_{n-1}, s_{n}\right]$. Nor can the minimum occur at $x=0$, for $q_{n}(0)=0$ and $q_{n}^{\prime}(0) \geq 0$ (since $\left.q_{n} \geq 0\right)$, nor at $x=s_{n-1}$, for then

$$
0 \geq p_{n}^{\prime}\left(s_{n-1}-0\right)=\left(q_{n}\left(s_{n-1}\right)-q_{n-1}\left(s_{n-1}\right)\right) / b-c\left(s_{n-1}\right) q_{n}\left(s_{n-1}\right),
$$

which implies that $q_{n}\left(s_{n-1}\right)=q_{n}^{\prime}\left(s_{n-1}\right)=p_{n}\left(s_{n-1}\right)=0$ since $q_{n} \geq 0$. Hence, 
$p_{n}(x)$ takes its negative minimum value at some point $x_{0} \in\left(0, s_{n-1}\right)$. On this interval, we have

$$
\begin{aligned}
\frac{p_{n}(x)-p_{n-1}(x)}{b} & =a(x) \frac{q_{n}^{\prime}(x)-q_{n-1}^{\prime}(x)}{b}+b(x) \frac{q_{n}(x)-q_{n-1}(x)}{b} \\
& =a(x)\left(L_{0} q_{n}\right)^{\prime}(x)+b(x)\left(L_{0} q_{n}\right)(x) \\
& =a(x)\left[p_{n}^{\prime}(x)+c(x) q_{n}(x)\right]^{\prime}+b(x)\left[p_{n}^{\prime}(x)+c(x) q_{n}(x)\right] \\
& =\left(L_{0} p_{n}\right)(x)+a(x) c^{\prime}(x) q_{n}(x) .
\end{aligned}
$$

Since $L_{0} p_{n}\left(x_{0}\right) \geq 0$, it follows that

$$
\begin{aligned}
\inf _{\left[0, s_{n}\right]}^{\operatorname{ing}} p_{n}(x) & =p_{n}\left(x_{0}\right) \geq p_{n-1}\left(x_{0}\right)-A C^{\prime} M_{0} b \\
& \geq \inf _{\left[0, s_{n-1}\right]} p_{n-1}(x)-A C^{\prime} M_{0} b
\end{aligned}
$$

where $C^{\prime}=\max \left[0, s_{\infty}\right] \mid c^{\prime} \%$. By induction, we obtain

(3.10) $\inf _{\left[0, s_{n}\right]} p_{n}(x) \geq \inf _{[0, d]} p_{0}(x)-A C^{\prime} M_{0} T \equiv-M_{2}(T) \quad(n=0,1,2, \cdots,[T / b])$.

This completes the proof.

Now let us define a curve $x=s^{b}(t)$ by setting $s^{b}(n b)=s_{n}(n=0,1,2, \ldots)$ and determining $s^{b}(t)$ for $n b<t<(n+1) b$ from the equation

$$
G\left(s^{b}(t)\right)=\frac{t-n b}{b} G\left(s_{n+1}\right)+\frac{(n+1) b-t}{b} G\left(s_{n}\right) .
$$

Then, by the last lemma, the function $G\left(s^{b}(t)\right)$ is Lipschitz continuous on every interval $0 \leq t \leq T$ with Lipschitz constant $M_{2}(T)$. Let $C_{b}$ be the approximate continuation region

$$
C_{b}=\left\{(x, t) ; 0<x<s^{b}(t), 0<t<\infty\right\}
$$

and define a function $u^{b}(x, t)$ on $C_{b}$ by

$$
u^{b}(x, t)=\frac{t-n b}{b} u_{n+1}(x)+\frac{(n+1) b-t}{b} \hat{u}_{n}(x)
$$

if $n b \leq t<(n+1) b$. We will show that $\left\{s^{b}, u^{b}\right\}$ converges to a solution $\{s, u\}$ of (1.2)-(1.7) as $b \rightarrow 0$. The necessary estimates are given in the next lemma.

Lemma 6. Let $\left\{s^{b}, u^{b}\right\}$ be defined as above, and let (1.9) be satis/ied. Then (a) for each $T>0$ there exists a constant $M_{3}(T)$ such that

$$
d \leq s^{b}(t)<s_{\infty} \quad(0 \leq t<\infty)
$$


and

$$
0 \leq s^{b}\left(t_{2}\right)-s^{b}\left(t_{1}\right) \leq M_{3}(T)\left(t_{2}-t_{1}\right)^{\gamma} \quad\left(0 \leq t_{1} \leq t_{2} \leq T\right),
$$

where $\gamma=(1+\beta)^{-1} \in[1 / 2,1)$;

(b) for $n b<t<(n+1) b$,

$$
0 \leq \partial u^{b}(x, t) / \partial t=q_{n+1}(x) \leq M_{0} \quad\left(0 \leq x \leq s^{b}(t)\right) ;
$$

(c) for all $(x, t) \in C_{b},|u(x, t)| \leq M$ and

$$
0 \leq L_{0} u^{b} \leq M_{0}
$$

(d) for all $(x, t) \in C_{b}$,

$$
\left|\partial u^{b} / \partial x\right| \leq M_{4}
$$

where $M_{4}=\max \left(M_{1}, \sup \left[d, s_{\infty}\right]\left|\psi^{\prime}\right|\right)$;

(e) for each $T>0$ there exists a constant $M_{5}(T)$ sucb that for all $\delta>0$ we bave

$$
\begin{aligned}
0 & \leq\left(u^{b}(x, t+\delta)-u^{b}(x, t)\right) / \delta \\
& \leq M_{s}(T)(1+2 b / \delta)\left[\left(s^{b}(t)-x\right)+M_{3}(T)(\delta+2 b)^{\gamma}\right]
\end{aligned}
$$

whenever $(x, t) \in C_{b}$ and $t+\delta \leq T-b$.

Proof. (a) Since $G$ is strictly increasing, so are $s^{b}(t)$ and $G\left(s^{b}(t)\right)$. Then

$$
\begin{aligned}
M_{2}(T) & \geq \frac{G\left(s^{b}\left(t_{2}\right)\right)-G\left(s^{b}\left(t_{1}\right)\right)}{t_{2}-t_{1}}=-\frac{1}{t_{2}-t_{1}} \int_{s^{b}\left(t_{1}\right)}^{s^{b}\left(t_{2}\right)}\left(L_{0} \psi\right)(\xi) d \xi \\
& \geq \frac{1}{t_{2}-t_{1}} \int_{s^{b}\left(t_{1}\right)}^{s^{b}\left(t_{2}\right)} c_{0}(\xi-d)^{\beta} d \xi=\frac{c_{0} \gamma}{t_{2}-t_{1}}\left[\left(s^{b}\left(t_{2}\right)-d\right)^{1+\beta}-\left(s^{b}\left(t_{1}\right)-d\right)^{1+\beta}\right] \\
& \geq \frac{c_{0} \gamma}{t_{2}-t_{1}}\left[s^{b}\left(t_{2}\right)-s^{b}\left(t_{1}\right)\right]^{1+\beta} \geq 0 .
\end{aligned}
$$

Setting $M_{3}(T)=\left(M_{2}(T) / c_{0} \gamma\right)^{\gamma}$, we obtain (3.13). (3.12) is obvious.

(b)-(d) follow immediately from (3.11) and Lemma 4.

(e) Choose $n$ and $k$ such that

$$
(n-1) b \leq t<n b<\cdots<(n+k-1) b<t+\delta \leq(n+k) b .
$$


Then by (3.14) we have

$$
\begin{aligned}
& u^{b}(x, t+\delta)-u^{b}(x, t) \\
& \quad=(t-(n-1) b) q_{n}(x)+\sum_{j=1}^{k-1} q_{n+i}(x) b+(t+\delta-(n+k-1) b) q_{n+k}(x) .
\end{aligned}
$$

Now $q_{m}\left(s_{m}\right)=0$ and $q_{m}^{\prime}(x) \geq a_{0}^{-1}$ [inf $\left.p_{m}-B M_{0}\right]$, so that, by Lemma 5 ,

$$
q_{m}(x)=-\int_{x}^{s_{m}} q_{m}^{\prime}(\xi) d \xi \leq M_{s}(T)\left(s_{m}-x\right)
$$

where $M_{5}(T)=a_{0}^{-1}\left(M_{2}(T)+B M_{0}\right)$. Hence,

$$
\begin{aligned}
u^{b}(x, t+\delta)-u^{b}(x, t) & \leq M_{s}(T)\left[\begin{array}{r}
(t-(n-1) b)\left(s_{n}-x\right)+\sum_{j=1}^{k-1} b\left(s_{n+j}-x\right) \\
+(t+\delta-(n+k-1) b)\left(s_{n+k}-x\right)
\end{array}\right] \\
& \leq M_{s}(T)(k+1) b\left(s_{n+k}-x\right) \leq M_{s}(T)(\delta+2 b)\left(s_{n+k}-x\right) .
\end{aligned}
$$

But

$$
s_{p+k}-s^{b}(t) \leq M_{3}(T)[(n+k) b-t]^{\gamma} \leq M_{3}(T)(\delta+2 b)^{\gamma},
$$

so that

$$
u^{b}(x, t+\delta)-u^{b}(x, t) \leq M_{5}(T)(\delta+2 b)\left[\left(s^{b}(t)-x\right)+M_{3}(T)(\delta+2 b)^{\gamma}\right],
$$

yielding (3.17).

Lemma 7. Let the bypotheses of Lemma 6 be satisfied. Then there exists a sequence $b_{m} \rightarrow 0$, a continuous function $s(t)$ defined on $[0, \infty)$, and a function $u(x, t)$ which is defined on the closure of the set $C=\{(x, t) \in Q ; 0<x<$ $s(t)\}$ and satisfies a uniform Lipschitz condition there, sucb that

(a) $s^{b} m(t) \rightarrow s(t)$ uniformly on compact sets,

(b) $s(t)$ satisfies the inequalities

$$
d \leq s(t) \leq s_{\infty} \quad(0 \leq t<\infty)
$$

and

$$
0 \leq s\left(t_{2}\right)-s\left(t_{1}\right) \leq M_{3}(T)\left(t_{2}-t_{1}\right)^{\gamma} \text { if } 0 \leq t_{1} \leq t_{2} \leq T,
$$

(c) if $\delta, \epsilon>0$, there exists an integer $m_{0}$ such that $K_{\delta} \subseteq C_{b_{m}}$ and $\left|u^{b}(x, t)-u(x, t)\right|<\epsilon \quad$ for $(x, t) \in K_{\delta}$ 
whenever $m \geq m_{0}$, where $K_{\delta}=\left\{(x, t) \in \bar{Q} ; 0 \leq x \leq s(t)-\delta, 0 \leq t \leq \delta^{-1}\right\}$.

Proof. By (3.12) and (3.13), the family $\left\{s^{b}(t)\right\}$ is uniformly bounded and equicuntinuous on compact sets. Hence, by the Arzela-Ascoli theorem together with Cantor's diagonal process, a sequence $b_{m} \rightarrow 0$ may be chosen so that (a) holds. (b) then follows immediately from (3.12) and (3.13). It is also clear that $K_{\delta} \cong C_{b_{m}}$ if $m$ is sufficiently large. For such $m,(3.14)$ and (3.16) imply that $u^{b} m(x, t)$ satisfies a Lipschitz condition on $K_{\delta}$ with Lipschitz constant $\max \left(M_{0}, M_{4}\right)$. Hence, by the Arzela-Ascoli theorem together with a diagonal process, we may replace $\left\{b_{m}\right\}$ with a subsequence, also denoted $\left\{b_{m}\right\}$, such that $u^{b_{m}}$ converges uniformly on each $K_{\delta}$ to a limit function $u$ which satisfies a Lipschitz condition on $\bigcup_{\delta>0} K_{\delta}$. $u$ may then be extended to the closure of $C$ while preserving the Lipschitz condition.

Lemma 8. Let $\{s, u\}$ be as defined in Lemma 7. Then $u$ satisfies (1.2) in C. Also, u satisfies (ii) of Definition 1.

Proof. By the standard theory of weak solutions, if we prove that

$$
\iint_{C} u L^{*} \phi d x d t=0, \text { all } \phi \in C_{0}^{\infty}(C)
$$

where $L^{*}$ is the formal adjoint of $L$, it will follow that $u$ satisfies (1.2) in $C$, together with (ii) of Definition 1. To prove (3.20), let $\delta>0$ and consider values of $b, T$ such that supp $\phi \subseteq K_{\delta} \cap Q_{T-b} \subseteq K_{\delta / 2} \subseteq \bar{C}_{b}$ and $2 M_{3}(T) b^{\gamma}<\delta$. Then

$$
\iint_{C} u^{b} L^{*} \phi d x d t=\sum_{n=0}^{N-1} \int_{n b}^{(n+1) b} \int_{0}^{s} n\left(L u^{b}\right) \phi d x d t
$$

where $N=[T / b]$, for $K_{\delta} \cap\left[s_{n}, \infty\right) \times[n h,(n+1) b]=\varnothing$. From (3.11) we obtain

$$
\begin{aligned}
\iint_{C} u^{b} L^{*} \phi d x d t \\
=\sum_{n=0}^{N-1} \int_{0}^{s} n \int_{n b}^{(n+1) b}\left[L_{0} u_{n}\left(n+1-\frac{t}{b}\right)-L_{0} u_{n+1}\left(n-\frac{t}{b}\right)-\frac{u_{n+1}-u_{n}}{b}\right] \phi(x, t) d t d x \\
=\sum_{n=0}^{N-1} \int_{0}^{s} L_{0}\left(u_{n}-u_{n+1}\right)\left[\int_{n b}^{(n+1) b}\left(n+1-\frac{t}{b}\right) \phi(x, t) d t\right] d x \\
=\sum_{n=0}^{N-1} \int_{0}^{s} L_{n-1} L_{0} \int_{n b}^{(n+1) b}\left(n+1-\frac{t}{b}\right)[\phi(x, t)-\phi(x, t-b)] d t d x \\
\quad+\sum_{n=0}^{N-1} \int_{s_{n-1}}^{s_{n}} L_{0} u_{n} \int_{n b}^{(n+1) b}\left(n+1-\frac{t}{b}\right) \phi(x, t) d t d x
\end{aligned}
$$


where we set $\phi \equiv 0$ outside $C$. Therefore,

$$
\left|\iint_{C} u^{b} L^{*} \phi d x d t\right| \leq 1 / 2 s_{\infty} M_{0} T b \max \left|\phi_{t}\right|+M_{0} M_{3}(T) b^{\gamma} \max |\phi| .
$$

Now set $b=b_{m}$ with $m \rightarrow \infty$. Since $u^{b} \rightarrow u$ uniformly on supp $\phi$ the estimate (3.21) implies (3.20).

Lemma 9. Let $\{s, u\}$ be as defined in Lemma 7. Then (1.3)-(1.6) are satisfied. Also, (1.7) is satisfied in the sense that

$$
\lim _{\delta \rightarrow 0} u_{x}(s(t)-\delta, t)=\psi^{\prime}(s(t)) \quad(0 \leq t<\infty) .
$$

Proof. (1.3)-(1.5) are satisfied trivially since they are satisfied for each $u^{b}$. To prove (1.6), let $\epsilon>0$ be arbitrary, and let $\left(x_{0}, t_{0}\right) \in C$. Choose $b=b_{m}$ with $m$ so large that $\left(x_{0}, t_{0}\right) \in C_{b}$ and

$$
\left|u\left(x_{0}, t_{0}\right)-u^{b}\left(x_{0}, t_{0}\right)\right|+\left|\psi\left(s^{b}\left(t_{0}\right)\right)-\psi\left(s\left(t_{0}\right)\right)\right|<2 \epsilon / 3 .
$$

Then

$$
\left|u\left(x_{0}, t_{0}\right)-\psi\left(s\left(t_{0}\right)\right)\right|<\left|u^{b}\left(x_{0}, t_{0}\right)-\psi\left(s^{b}\left(t_{0}\right)\right)\right|+2 \epsilon / 3 \text {. }
$$

If $t_{0}=(n+\theta) b, \theta \in[0,1)$, and if $m$ is so large that $\left|x_{0}-s^{b}\left(t_{0}\right)\right| \leq 2 \delta$ where $\delta=\left|s\left(t_{0}\right)-x\right|$, then

$$
\begin{aligned}
\left|u^{b}\left(x_{0}, t_{0}\right)-\psi\left(s^{b}\left(t_{0}\right)\right)\right| \leq & \left|\hat{u}_{n}(x)-\psi\left(s_{n}\right)\right|(1-\theta)+\left|u_{n+1}\left(x_{0}\right)-\psi\left(s_{n+1}\right)\right| \theta \\
& +\left|\psi\left(s^{b}\left(t_{0}\right)\right)-(1-\theta) \psi\left(s_{n}\right)-\theta \psi\left(s_{n+1}\right)\right| \\
& \leq M_{4}\left\{(1-\theta)\left[\left|x_{0}-s_{n}\right|+\left|s^{b}\left(t_{0}\right)-s_{n}\right|\right]+\theta\left[\left|x_{0}-s_{n+1}\right|+\left|s^{b}\left(t_{0}\right)-s_{n+1}\right|\right]\right\} \\
& \leq M_{4}\left[\left|x_{0}-s^{b}\left(t_{0}\right)\right|+2(1-\theta)\left|s^{b}\left(t_{0}\right)-s_{n}\right|+2 \theta\left|s^{b}\left(t_{0}\right)-s_{n+1}\right|\right]
\end{aligned}
$$

by (3.11) and Lemma 6, whence

$$
\left|u^{b}\left(x_{0}, t_{0}\right)-\psi\left(s\left(t_{0}\right)\right)\right| \leq 2 M_{4}\left(\delta+M_{3}(T) b^{\gamma}\right) \quad \text { if } 0 \leq t_{0} \leq T .
$$

If $m$ is so large that $3 M_{3}(T) b^{\gamma}<\epsilon$, we obtain $\left|u\left(x_{0}, t_{0}\right)-\psi\left(s\left(t_{0}\right)\right)\right|<\epsilon+2 M_{4} \delta$, whence we conclude that

$$
\left|u\left(x_{0}, t_{0}\right)-\psi\left(s\left(t_{0}\right)\right)\right| \leq 2 M_{4} \delta .
$$

In view of the continuity of $u$ on $\bar{C}$, this establishes (1.6).

In any compact subset of $\left[0, s\left(t_{0}\right)\right), u_{x}^{b}\left(x_{,} t_{0}\right)$ is defined and continuous if $b$ is sufficiently small. By (3.15) and (3.16), these functions are uniformly bounded 
and equicontinuous there. Hence, by restricting $b$ to a subsequence of $\left\{b_{m}\right\}$, we may arrange that $u_{x}^{b}\left(x, t_{0}\right) \rightarrow u_{x}\left(x, t_{0}\right)$ uniformly on compact subsets. By (3.15), $u_{x}$ then satisfies a Lipschitz condition in the $x$-variable which is uniform on $C$. Hence, $u_{x}$ may be extended to $\partial C$ via its tangential boundary limits. Proceeding as above, we may derive an inequality similar to (3.23) which will establish (3.22).

Lemma 10. Let $\{s, u\}$ be as defined in Lemma 7. Then

(a) $s(t)$ is Lipschitz continuous for $t>0$.

(b) If $u_{x}$ is defined on $\bar{C}$ as in the proof of Lemma 9, then $u_{x}$ is continuous on $\bar{C}$. Moreover, (1.7) bolds.

(c) $u_{t^{\prime}}$ and therefore $u_{x x^{\prime}}$, has a continuous extension to $\bar{C}-(0,0)$.

Proof. Since Lemmas 8 and 9 provide all the information required in the proof of Lemma 1 and $s(t)$ is nondecreasing, we have $s(t)>d$ for $0<t<\infty$. If $t_{0}>0$, let $\epsilon=\left(s\left(t_{0}\right)-d\right) / 2$ and choose $\delta>0$ such that $s(t) \geq d+\epsilon$ for $t_{0}-\delta \leq$ $t \leq t_{0}+\delta$. Let $C(\epsilon)=\inf _{\xi \geq d+\epsilon}\left[-L_{0} \psi(\xi)\right]>0$. Then, since $G\left(s^{b}(t)\right)$ satisfies a Lipschitz condition with Lipschitz constant $M_{2}(T)$ on $0 \leq t \leq T$, the same is true of $G(s(t))$ and we have

$$
\begin{aligned}
M_{2}(T) & \geq\left|\frac{G(s(t))-G\left(s\left(t_{0}\right)\right)}{t-t_{0}}\right|=\frac{1}{\left|t-t_{0}\right|} \int_{s\left(t \wedge t_{0}\right)}^{s\left(t \vee t_{0}\right)}\left[-L_{0} \psi(\xi)\right] d \xi \\
& \geq C(\epsilon)\left|\frac{s(t)-s\left(t_{0}\right)}{t-t_{0}}\right| \quad\left(t_{0}-\delta \leq t \leq t_{0}+\delta\right) .
\end{aligned}
$$

Hence, $\left|s(t)-s\left(t_{0}\right)\right| \leq C(\epsilon)^{-1} M_{2}(T)\left(t-t_{0}\right)\left(t_{0}-\delta \leq t \leq t_{0}+\delta\right)$ which proves (a). Now $u_{x}$ and $u_{t}$ are in the class $C^{2,1}(C)$ and therefore satisfy parabolic equations in $C$. By Lemma 9, $u_{x}$ has continuous tangential boundary values up to the Lipschitz curve $x=s(t)$. But it is easy to show by the classical methods of Gevrey [7] that a solution of a parabolic equation which has continuous tangential boundary values up to a Lipschitz boundary curve $x=s(t)$ is continuous up to that curve. The rest of (b) follows from standard facts. To prove (c), let $b=b_{m}$ with $m \rightarrow \infty$ in (3.17). We obtain

$$
0 \leq(u(x, t+\delta)-u(x, t)) / \delta \leq M_{5}(T)\left[(s(t)-x)+\delta^{\gamma}\right]
$$

for $(x, t) \in \bar{C}$ with $0 \leq t \leq T$. Letting $\delta \rightarrow 0$ yields $0 \leq u_{t}(x, t) \leq M_{s}(T)(s(t)-x)$ in $\bar{C} \cap Q_{T}$. Hence, $u_{t}$ has tangential boundary values which vanish identically on $x=s(t)$. (c) now follows in the same way as (b).

We summarize the results of Lemmas $7-10$ as 
Theorem 6. Let (1.9) be satisfied. Then there exists a solution $\{s, u\}$ of problem (1.2)-(1.7). Moreover, if $\hat{u}$ is defined by $(1.8)$, then $\hat{u}_{t}$ and $\hat{u}_{x}$ are Hölder continuous except at the origin.

The last statement of the theorem is obvious.

Remark. In view of the proof of Lemma 10(a), the assumption that (1.9) holds for all $x \geq d$ may be considerably weakened. It is necessary only to suppose that (1.9) holds for $d \leq x \leq d+\epsilon$ for some $\epsilon>0$ and that (2.2) is satisfied.

Added in proof. Since the submission of this paper, the author has learned of two recent developments which should be mentioned here. (i) P. Van Moerbeke, in a thesis written at The Rockefeller University, has solved an optimal stoppingfree boundary problem by treating an equivalent Volterra integral equation. His initial condition differs from our (1.5), and satisfies a compatibility assumption which makes the free boundary curve turn out to be Lipschitz near $t=0$. He also treats a number of specific cases not covered by his general theorem. (ii) A. Bensoussan and J. L. Lions have discussed the equivalence of an optimal stopping problem for a diffusion on $R^{n}$ with a parabolic variational inequality, which is a weak formulation of the minimum problem in our Theorem 1. They prove existence, uniqueness, etc. for the latter problem.

\section{REFERENCES}

1. H. Chernoff, Sequential tests for the mean of a normal distribution, Proc. Fourth Berke ley Sympos. Math. Statist. and Probability, vol. 1, Univ, of California Press, Berkeley, Calif., 1961, pp. 79-92.

2. -...... Optimal stochastic control, Sankhyā Ser. A 30 (1968), 221-252. MR 39 \#2494.

3. E. B. Dynkin, Markov processes, Fizmatgiz, Moscow, 1963; English transl., Vols. I, II, Die Grundlehren der math. Wissenschaften, Bände 121, 122, Academic Press, New York; Springer-Verlag, Berlin, 1965. MR 33\#1886; $33 \# 1887$.

4. - The optimum choice of the instant for stopping a Markov process, Dokl. Akad. Nauk SSSR 150 (1963), 238-240 = Soviet Math. Dokl. 4 (1963), 627-629. MR 27 \#4278.

5. E. B. Dynkin and A. A. Juškevič, Markov processes; Theorems and problems, "Nauka", Moscow, 1967; English transl., Plenum Press, New York, 1969. MR 36 \#6006; $39 \# 3585$.

6. A. Friedman, Interior estimates for parabolic systems of partial differential equa. tions, J. Math. Mech. 7 (1958), 393-417. MR 21 \#362.

7. M. Gevrey, Sur les équations aux dérivées partielles du type parabolique, J. Math. 9 (6) (1913), 305-471.

8. B. I. Grigelionis, Optimal stopping of stochastic processes, Proc. Sixth Math. Summer School: Prob. Theory and Math. Statist. (Kačivili, 1968), Akad. Nauk Ukrain. SSR Inst. Mat., Kiev, 1969, pp. 176-210. (Russian) MR 43 \#2761.

9. B. I. Grigelionis and A. N. Širjaev, On the Stefan problem and optimal stopping 
rules for Markov processes, Teor. Verojatnost. i Primenen. 11 (1966), 612-631 = Theor. Probability Appl. 11 (1966), 541-558. MR 35 \#7538.

10. K. Itô, Lectures on stochastic processes, Tata Institute of Fundamental Research, Bombay 1961.

11. I. I. Kolodner, Free boundary problem for the heat equation with applications to problems of change of phase. I. General method of solution, Comm. Pure Appl. Math. 9 (1956), 1-31. MR 19, 285.

12. S. N. Kružkov, On some problems with unknown boundaries fọ the heat conduction equation, Prikl. Mat. Meh. 31 (1967), 1009-1014 = J. Appl. Math. Mech. 31 (1967), 1014-1024. MR 37 \#6618.

13. H. P. Mc Kean, Jro, $A$ free boundary problem for the heat equation arising from a a problem of mathematical economics, Industrial Management Review 6 (1965), 13-39; reprinted in The Random Character of Stock Market Prices, P. Cootner (ed.), M.I.T. Press, Cambridge, Mass., 1964, pp. 525-532.

14. E. Röthe, Zweidimensionale parabolische Randwertaufgaben als Grenzfall eindimensionaler Randwertaufgaben, Math. Ann. 102 (1930), 650-670.

15. G. G. Sackett, An implicit free boundary problem for the heat equation, SIAM J. Numer. Anal. 8 (1971), 80-96. MR 44 \#654.

16. $\longrightarrow$ Numerical solution of a parabolic free boundary problem arising in statis tical decision theory, Math. Comp. 25 (1971), 425-434.

17. B. Sherman, Some comments on free boundary problems for parabolic equations arising in statistical decision theory, Rocketdyne Res. Report \#66-24, 1966.

18. N. D. Thi, On a free boundary problem for a parabolic equation, Vestnik Moskov. Univ. 2 (1966), 40-54. (Russian)

19. T. D. Ventcel', A free boundary problem for the heat equation, Dokl. Akad. Nauk SSSR 131 (1960), 1000-1 003 = Soviet Math. Dokl. 1 (1960), 358-361. MR 22 \#8225.

DEPARTMENT OF MATHEMATICS, RICE UNIVERSITY, HOUSTON, TEXAS 77001

Current address : Department of Mathematics, University of Kentucky, Lexington, Kentucky 40506 